\title{
Two-beam coupling by nitrogen-vacancy centers in diamond
}

\author{
D. Redman, Q. Shu, A. Lenef, and S. C. Rand \\ Division of Applied Physics, Department of Electrical Engineering and Computer Science, \\ 1301 Beal Avenue, University of Michigan, Ann Arbor, Michigan 48109-2122
}

Received September 17, 1991

\begin{abstract}
A new method for two-beam coupling measurements based on acousto-optic modulation with subhertz tuning capability is applied to study excited-state dynamics of the nitrogen-vacancy center in diamond. Results yield fundamental decay constants and the nonlinear refractive index and are in good agreement with the calculated dual grating response to resonances in the microscopic susceptibility due to intersystem crossing and spinlattice relaxation.
\end{abstract}

In recent years two-beam coupling has become established as a precise method for investigating nonlinear refractive indices of saturable absorbers. ${ }^{1}$ Intersecting laser beams with finite detuning are typically used to generate slowly moving optical gratings in absorptive solids with phases that lead those of refractive-index changes caused by slow excited-state dynamics. Energy transfer between pump and probe waves then occurs, with detuningdependent gain. This method has been employed to study nonlinearities in $\mathrm{Cr}$-doped laser materials ${ }^{2}$ with simple impurity energy levels and hostdependent nonlinearities of relevance to high-power tunable Cr-laser performance.

Here we introduce a method that furnishes twobeam coupling spectra versus detuning in seconds, and we use it to study simple impurity centers in diamond. With it, relaxation processes of the nitrogen-vacancy $(\mathrm{N}-\mathrm{V})$ color center, for which the energy-level structure was determined only recently, ${ }^{3}$ are characterized. By extending two-beam coupling theory to incorporate two separate decay processes contributing to saturation, we obtain good agreement between complex changes in signal intensity and detuning, as well as consistency with earlier determinations of N-V relaxation times. We also report estimates of real and imaginary parts of the nonlinear index.

The N-V center consists of a N-V complex in diamond, ${ }^{4}$ one of many point defects ${ }^{5}$ that need to be characterized carefully to evaluate potential applications of this emerging wide-gap semiconductor. The electronic structure ${ }^{3}$ and absorption spectrum of the center are shown in Fig. 1(a). As indicated by three-pulse photon echo data in Fig. 1(b), the ${ }^{3} E$ state decays rapidly in $13.3 \pm 0.2 \mathrm{~ns}$, revealing that this decay channel cannot contribute to optical saturation at low intensities. ${ }^{6}$ However, intersystem crossing from the ${ }^{1} A$ to the ground state $(\tau=$ $265 \mathrm{~ms}$ ) and the spin-lattice relaxation between the ${ }^{3} A$ ground-state components $(\tau=1.17 \mathrm{~ms})$ are extremely slow. ${ }^{3}$ Hence in the N-V center we expect two physically distinct processes to contribute to low-intensity saturation behavior at wavelengths near the first resonance, unlike the single- component saturation in Cr-doped solids. Moreover, the response times of these contributions should have a one-to-one correspondence with resonances observed in nearly degenerate four-wave mixing (NDFWM), which probes the microscopic susceptibility directly. ${ }^{3}$

To account for contributions from multiple components, we extended previous expressions ${ }^{1}$ for pump and probe wave intensities $I_{1}$ and $I_{2}$, respectively, as follows:

$$
\begin{aligned}
& \frac{\mathrm{d}}{\mathrm{d} z} I_{1}=\left(g_{I}-g_{R}\right) I_{1} I_{2}+g\left(I_{1}+I_{2}\right) I_{1}-\alpha I_{1}, \\
& \frac{\mathrm{d}}{\mathrm{d} z} I_{2}=\left(g_{I}+g_{R}\right) I_{1} I_{2}+g\left(I_{1}+I_{2}\right) I_{2}-\alpha I_{2} .
\end{aligned}
$$

Here $g_{R}=\beta n_{2}{ }^{\prime}\left(p_{1} \sin \phi_{1}+p_{2} \sin \phi_{2}\right)$ and $g_{I}=$ $\beta n_{2}{ }^{\prime \prime}\left(p_{1} \cos \phi_{1}+p_{2} \cos \phi_{2}\right)$ are real and imaginary gain coefficients, respectively, and $g=\beta n_{2}{ }^{\prime \prime} . \quad \beta=$ $2 \pi / \lambda \cos (\theta / 2)$, where $\theta$ is the interbeam angle. We assumed a Debye relaxation model with two additive contributions to nonlinear gain. Phase angles $\phi_{i}$ between optical and index gratings are determined by the correspondence $p_{i} \exp \left(i \phi_{i}\right)=A_{i}\left(1+i \Omega \tau_{i}\right)^{-1}$, where $A_{i}$ and $p_{i}$ are real constants, $\Omega=\omega_{2}-\omega_{1}$, and $\tau_{i}$ is the Debye decay time governing saturation process $i$. These equations have been solved previously for a single response time in the case of $g_{I}=0$. When several decay processes make important contributions to system saturation simultaneously, and $g_{I}$ is not zero, they still admit a simple solution for weak probe intensity $\left(I_{2} \ll I_{1}\right)$ :

$$
\begin{aligned}
I_{2}(z) & =I_{2}(0) e^{-\alpha z}\left[1-g I_{1}(0) z\right]^{-\Gamma} \\
& \cong I_{2}(0) e^{-\alpha z}\left[1+\Gamma g I_{1}(0) z\right],
\end{aligned}
$$

where

$$
\Gamma \equiv 1+\frac{A}{r}\left(\frac{r-\Omega \tau_{1}}{1+\Omega^{2} \tau_{1}^{2}}\right)+\frac{B}{r}\left(\frac{r-\Omega \tau_{2}}{1+\Omega^{2} \tau_{2}^{2}}\right) .
$$

Here $n_{2}=n_{2}{ }^{\prime}+i n_{2}{ }^{\prime \prime}, r=n_{2}{ }^{\prime \prime} / n_{2}{ }^{\prime}$, and $A$ and $B$ are (intensity-dependent) constants. From Eq. (3) it becomes apparent that when $n$ dynamic decay processes govern saturation recovery, $n$ dispersive and absorptive components contribute to two-beam coupling gain. 


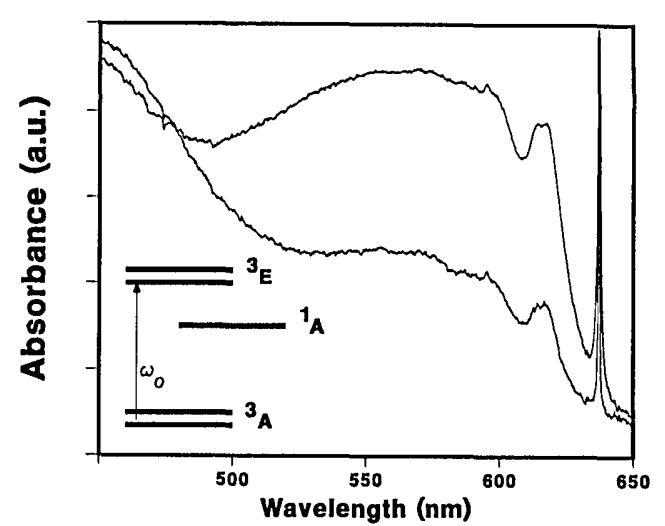

(a)

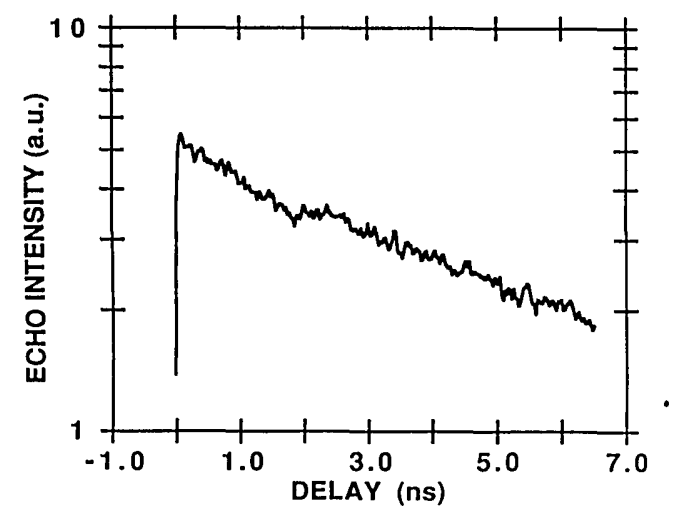

(b)

Fig. 1. (a) Unpolarized absorption spectra for annealed samples with 3.1 and $7.7 \times 10^{18} \mathrm{~N}-\mathrm{V} / \mathrm{cm}^{-3}$ from dosages of 2 and $5 \times 10^{17} \mathrm{e} / \mathrm{cm}^{2}$ (electron paramagnetic resonance calibration). Inset shows the N-V electronic structure. (b) Stimulated echo decay curve obtained at $637 \mathrm{~nm}$ with 800-fs pulses from a dual jet, synch-pumped DCM dye laser. Population decay time is twice the observed $\tau$. Accumulated echo contributions were negligible owing to weak illumination, low cavity-dumping rate, and high chopping frequency.

Saturation-induced changes in probe intensity, proportional to the gain coefficient $\Gamma$, were measured with a frequency-stabilized DCM ring dye laser at $637 \mathrm{~nm}$. As indicated in Fig. 2, two acousto-optic modulators were used to generate rf-tunable pump and probe beams with a relative intensity ratio of 10:1 and an external interbeam angle of $3^{\circ}$. Beams were linearly polarized along [001] and impinged on the (100) plane, bisected by [100]. Relative detuning of pump and probe frequencies was accomplished with a versatile combination of acousto-optic frequency and phase modulation. Probe frequency was tuned relative to the pump frequency by an integer number of hertz and by a variable, linear voltage ramp applied to the phase modulation input of the synthesizer for fine tuning from 0 to $1.0 \mathrm{~Hz}$. The signal-to-noise ratio was improved with vibration isolation and synchronous detection at a high chopping frequency to avoid modulation sidebands. ${ }^{7}$ Signals were verified to be independent of $\theta$ in the range $0-10^{\circ}$. Samples were prepared from type-Ib synthetic-diamond single crystals containing 220 parts in $10^{6}$ single substitutional nitrogen atoms using $10^{16}-10^{18} \mathrm{~cm}^{-2}$ doses of $1.7-\mathrm{MeV}$ electrons and subsequent annealing at $820^{\circ} \mathrm{C}$ for $4 \mathrm{~h}$ in argon. ${ }^{4}$
In Fig. 3, two-beam coupling signals from several different samples are shown. Figure 3(a) displays the predominantly dispersive spectral shapes obtained for $\mathrm{Cr}: \mathrm{Al}_{2} \mathrm{O}_{3}$ and $\mathrm{Cr}: \mathrm{YAlO}_{3}$. These spectra consist of ten signal-averaged scans acquired in $1 \mathrm{~min}$. Their spectral widths reflect the ratio of ${ }^{2} E$ decay times in these two hosts, ${ }^{8,9}$ namely 1:10. Consistent with earlier research, ${ }^{2}$ the fitting parameters for ruby are $\tau=3.1 \mathrm{~ms}$ and $n_{2}^{\prime \prime} / n_{2}^{\prime}=0.028$. The parameters for Cr:YAlO $\mathrm{YH}_{3}$ are $\tau=28.6 \mathrm{~ms}$ and $n_{2}{ }^{\prime \prime} / n_{2}{ }^{\prime}=-0.03$. Figure $3(\mathrm{~b})$ illustrates the different spectral signature observed in diamond. The solid curve gives the theoretical fit based on Eq. (3). The best-fitted parameters $(T=295 \mathrm{~K})$ are

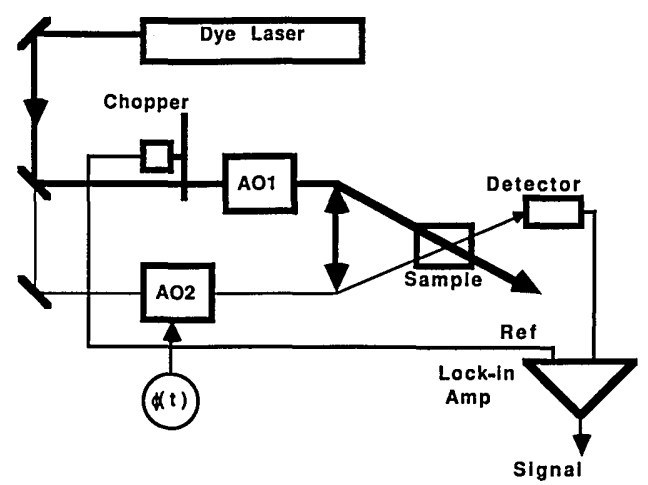

Fig. 2. Experimental apparatus, showing chopped pump and tuned probe beams. AO1 and AO2 are acousto-optic modulators driven by phase-locked oscillators tunable in frequency by single-hertz steps or subhertz increments by using phase modulation.

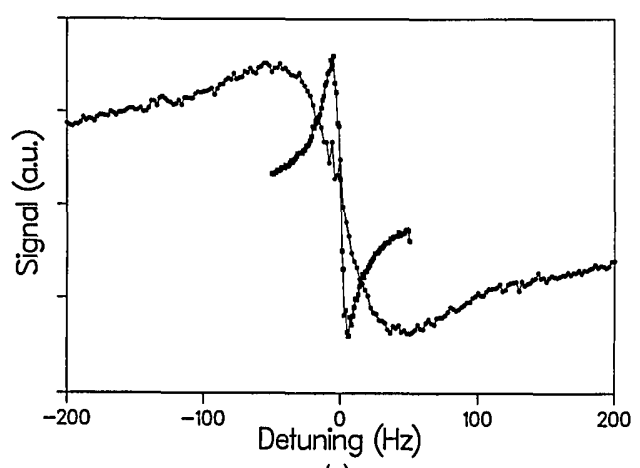

(a)

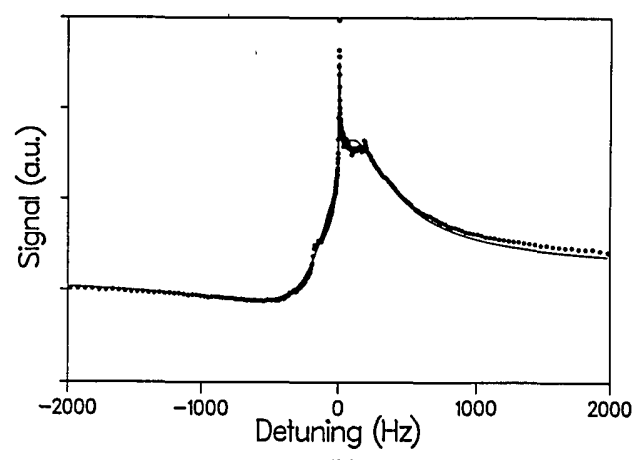

(b)

Fig. 3. (a) Two-beam coupling signals versus detuning in $\mathrm{Cr}: \mathrm{Al}_{2} \mathrm{O}_{3}$ (wide trace) and $\mathrm{Cr}: \mathrm{YAlO}_{3}$ (narrow trace). (b) Two-bcam coupling at $637 \mathrm{~nm}$ in synthetic diamond at room temperature $\left(7.7 \times 10^{18} \mathrm{~N}-\mathrm{V} / \mathrm{cm}^{3}\right)$. The solid curve is a nonlinear least-squares regression based on Eq. (3). 


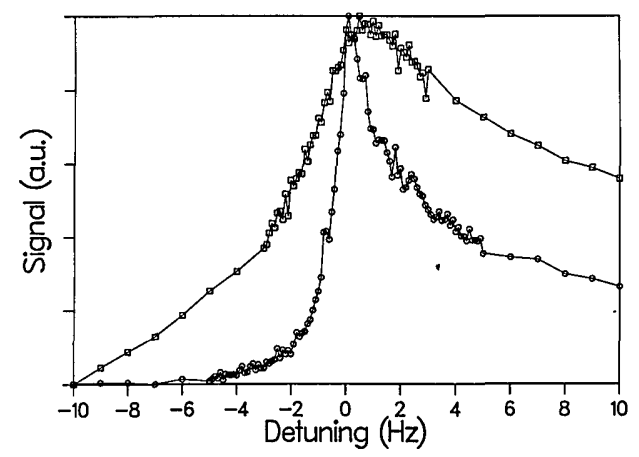

Fig. 4. Two-beam coupling signals versus detuning, illustrating subhertz resolution at $T=77 \mathrm{~K}$ (circles) and $T=295 \mathrm{~K}$ (squares). At small detunings $\Delta$, signals are time averaged for periods $\Delta^{-1}$.

$n_{2}^{\prime \prime} / n_{2}^{\prime}=-1.01 \pm 0.04, \tau_{1}=50 \pm 2 \mathrm{~ms}$, and $\tau_{2}=0.64 \pm 0.01 \mathrm{~ms}$.

The dual grating response incorporated into Eq. (3) accurately reproduces the structured, asymmetric shape of diamond spectra. Additionally, however, time constants may be compared quantitatively with precise NDFWM determinations. ${ }^{3}$ NDFWM values, available only at liquid-nitrogen temperature, are $\tau_{1}=265.3 \pm 0.6 \mathrm{~ms}$ and $\tau_{2}=$ $1.170 \pm 0.003 \mathrm{~ms}$. Beam coupling at $77 \mathrm{~K}$ gave $n_{2}{ }^{\prime \prime} / n_{2}^{\prime}=-0.9 \pm 0.1, \tau_{1}=92 \pm 30 \mathrm{~ms}$, and $\tau_{2}=1.1 \pm 0.1 \mathrm{~ms}$ from poorer (unweighted) fits than those obtained at room temperature. Good agreement is found for $\tau_{2}$, while $\tau_{1}$ is somewhat shorter than the NDFWM value. The long-lived $\tau_{1}$ component is particularly susceptible to power broadening and transient grating effects from mechanical chopping, and it is also uniquely sensitive to excited-state absorption. $\tau_{1}$ from beam coupling approaches the NDFWM value as intensity and chopping duty cycle are reduced, but only extreme experimental precautions and weighted fits achieve full agreement. Using knife-edge measurements of intensity profiles, we estimated real and imaginary parts of $n_{2}=n_{2}{ }^{\prime}+i n_{2}{ }^{\prime \prime}$ at $T=295 \mathrm{~K}$ to be $n_{2}{ }^{\prime \prime}=(1.5 \pm 0.6) \times 10^{-6} \mathrm{~cm}^{2} / \mathrm{W}$ and $n_{2}{ }^{\prime}=$ $(-1.7 \pm 0.4) \times 10^{-6} \mathrm{~cm}^{2} / \mathrm{W}$.

Details of the narrowest spectral features are determined by the slowest dynamics. In the case of the N-V center, this is the intersystem crossing process, in general strongly temperature-dependent in color centers. ${ }^{7,10}$ Consequently, it is not surprising that the results in Fig. 4 reveal a much narrower central feature with a correspondingly longer time constant at $77 \mathrm{~K}$ than at $295 \mathrm{~K}$. Points in the center of Fig. 4 illustrate the high resolution possible with phase modulation.

Power broadening was noticeable above intensities of $5 \mathrm{~W} / \mathrm{cm}^{2}$. Also, above $100 \mathrm{~W} / \mathrm{cm}^{2}$, a small increase in baseline absorption appeared. Since persistent spectral hole burning within the inhomogeneously broadened absorption line occurs only below liquidnitrogen temperature, ${ }^{11,12}$ this effect must reflect changes in the homogeneously broadened absorption of the center, possibly due to photoionization from an excited state. Indeed, direct measurements revealed weak photoconductive currents depending linearly on incident intensity in the N-V absorption band. Charge generation and the large negative value observed for $r$ (indicative of metastable excited-state absorption ${ }^{13}$ ) could arise from photoionization of the saturated ${ }^{1} A$ level if this level were within $1.945 \mathrm{eV}$ of the conduction-band edge.

In summary, there is good agreement between beam-coupling observations in diamonds and theory extended to account for intersystem crossing and spin-lattice dynamics. Each process makes a distinct contribution to $n_{2}$. The ratio $r=n_{2}{ }^{\prime \prime} / n_{2}{ }^{\prime}$, being large and negative, indicates strong excited-state absorption from the ${ }^{1} A$ metastable level, possibly to the conduction band.

This research was sponsored by Sumitomo Electric Company, the U.S. Air Force Office of Scientific Research, the Center for Ultrafast Optical Science, and a Phoenix Memorial Laboratory grant. We acknowledge technical support by S. Yazu, K Tsuji, S. Satoh, and L. E. Halliburton and discussions with N. B. Manson and R. N. Schwartz. D. Redman is grateful for AT\&T fellowship support.

\section{References}

1. Y. Silberberg and I. Bar-Joseph, J. Opt. Soc. Am. B 1, 662 (1984); M. A. Kramer, W. R. Tompkin, and R. W. Boyd, Phys. Rev. A 34, 2026 (1986); I. McMichael, P. Yeh, and P. Beckwith, Opt. Lett. 13, 500 (1988); S. A. Boothroyd, J. Chrostowski, and M. S. O'Sullivan, J. Opt. Soc. Am. B 6, 766 (1989).

2. S. A. Boothroyd, J. Chrostowski, and M. S. O'Sullivan, Opt. Lett. 14, 946 (1989); C. L. Adler and N. M. Lawandy, Opt. Commun. 81, 33 (1991); J. C. Penaforte, E. A. Gouveia, and S. C. Zilio, Opt. Lett. 16, 452 (1991).

3. N. B. Manson, X.-F. He, and P. T. H. Fisk, Opt. Lett. 15, 1094 (1990); D. Redman, S. Brown, R. H. Sands, and S. C. Rand, Phys. Rev. Lett. 67, 3420 (1991).

4. G. Davies and M. F. Hamer, Proc. R. Soc. London Ser. A 348, 285 (1976).

5. C. D. Clark, E. Mitchell, and B. Parsons, in The Properties of Diamond, J. E. Field, ed. (Academic, New York, 1979), pp. 23-77.

6. See also A. T. Collins, M. F. Thomaz, and M. I. Jorge, J. Phys. C 16, 2177 (1983).

7. S. C. Rand, Opt. Lett. 11, 135 (1986).

8. S. Kisliuk and C. A. Moore, Phys. Rev. 160, 307 (1967).

9. D. G. Steel, S. C. Rand, and J. Liu, J. Opt. Soc. Am. B 4, 1794 (1987).

10. Y. Farge, J. M. Ortega, and R. H. Silsbee, J. Chem. Phys. 69, 3972 (1978).

11. D. Redman, S. Brown, and S. C. Rand, "Origin of persistent hole burning of N-V color centers in diamond," J. Opt. Soc. Am. B (to be published).

12. R. T. Harley, M. J. Henderson, and R. M. Macfarlane, J. Phys. C 17, L233 (1984).

13. T. Catunda, J. P. Andreeta, and J. C. Castro, Appl. Opt. 25, 2391 (1986). 